\title{
THE
}

\section{Heisenberg Antiferromagnetic Chains: Quantum-Classical Crossover}

Jill C. Bonner

University of Rhode Island

Gerhard Müller

University of Rhode Island, gmuller@uri.edu

M. P. Nightingale

University of Rhode Island, nightingale@uri.edu

H. W.J. Blöte

J. B. Parkinson

Follow this and additional works at: https://digitalcommons.uri.edu/phys_facpubs

Terms of Use

All rights reserved under copyright.

\section{Citation/Publisher Attribution}

Jill C. Bonner, Gerhard Müller, M.P. Nightingale, H.W.J. Blöte and J.B. Parkinson. Heisenberg

antiferromagnetic chains: quantum-classical crossover. J. Magnetism and Magn. Materials 54-57 (1986), 1253-1254.

Available at: http://www.sciencedirect.com/science/article/pii/030488538690805X

This Article is brought to you for free and open access by the Physics at DigitalCommons@URI. It has been accepted for inclusion in Physics Faculty Publications by an authorized administrator of DigitalCommons@URI. For more information, please contact digitalcommons-group@uri.edu. 


\title{
Heisenberg Antiferromagnetic Chains: Quantum-Classical Crossover
}

\author{
Jill C. Bonner, ${ }^{1}$ Gerhard Müller, 1 M. P. Nightingale, 1 H. W. J. Blöte, ${ }^{2}$ and J. B. \\ Parkinson 3 \\ ${ }^{1}$ Department of Physics, University of Rhode Island, Kingston RI 02881, USA \\ 2 Technical University of Delft, Delft, The Netherlands \\ 3 UMIST, P.O. Box 88, Manchester M60 1QD, UK
}

\begin{abstract}
An unusual crossover mechanism has been discovered by numerical investigation of the dispersion spectrum of Heisenberg antiferromagnetic chains with various spin values in a magnetic field. This result is reflected in novel behavior of static properties such as the integrated intensity. A study of various excitation gaps using finite chain calculations extended by quantum Monte Carlo studies indicates unusual behaviour in the $T=0$ magnetization isotherms.
\end{abstract}

\section{Quantum-classical crossover}

We have undertaken a comprehensive numerical investigation of Heisenberg anti ferromagnetic (HBAFM) chains extending in size up to the following values of chain length $N$ and spin-value $S$, $(20,1 / 2)(14,1)(10,3 / 2)$ and $(8,2)$. Our motivation has been twofold: (1) Classical and quantum (spin-1/2) dispersion spectra in an applied magnetic field show striking differences. Perhaps the most striking is the presence of a soft mode which "tracks" across the Brillouin zone in the spin- $1 / 2$ case, a feature absent in the classical case. Hence the crossover from quantum to classical behavior is of great interest. (2) Haldane has made the surprising prediction [1] that integer-spin HBAFM's at $T=0$ show notably different phase behavior from half-integer-spin HBAFM's. This would indicate, inter alia, that our understanding of the simplest magnetic systems is incomplete. Our finite chain calculations rely on the Lanczos technique to obtain the two lowest-lying eigenvalues for all allowed values of $S_{T}^{z}$ and wave-vector $q$. The investigation for all values of $S_{T}^{z}$ is equivalent to investigation in a magnetic field extending from $H=0$ to the antiferromagnetic critical field $H_{\mathrm{c}}$ [2]. Two distinct regimes have been identified from the dispersion spectra for $S \geq 1$, one quantum in character as for $S=1 / 2$ and the other showing "classical behavior" [2]. As illustrated in fig. 1, the relative extent of, say, the quantum regime varies with $S$, vanishing as $1 / 2 S$ in the classical limit.

The quantum and classical features are not only apparent in the dispersion spectra, but in the bulk thermodynamic properties such as the $T=0$ magnetization isotherms as a function of field $[2,3]$ and in other static quantities such as the real-space correlation functions and their Fourier transforms, the integrated intensities [2.3]. The integrated intensities for $N=14, S=1$ for the various allowed q-values compared with the classical expression

$$
I^{z z}(q)=\frac{S}{2}\left\{1-\left(\frac{H}{H_{\mathrm{c}}}\right)^{2}\right\}\left[\frac{1-\cos q}{1+\left(1-2\left(H / H_{\mathrm{c}}\right)^{2}\right) \cos q}\right]^{1 / 2},
$$

evaluated at equivalent $q$-values are shown in fig. 2. The corresponding finite-chain plot for $S=1 / 2$ displays prominent cusps at the soft-mode $q$-values for the entire magnetization (or field) range. In the spin-1 plot, weak cusps are observed only in the magnetization range $M_{\mathrm{sat}} \geq M \geq M_{\mathrm{sat}}(1-$ $1 / 2 S$ ), which corresponds to the quantum regime of fig. 1 . No cusps are visible in the remaining 
range $M_{\text {sat }}(1-1 / 2 S)>M \geq 0$, in qualitative accordance with the corresponding classical (spin- $\left.\infty\right)$ intensity curves.

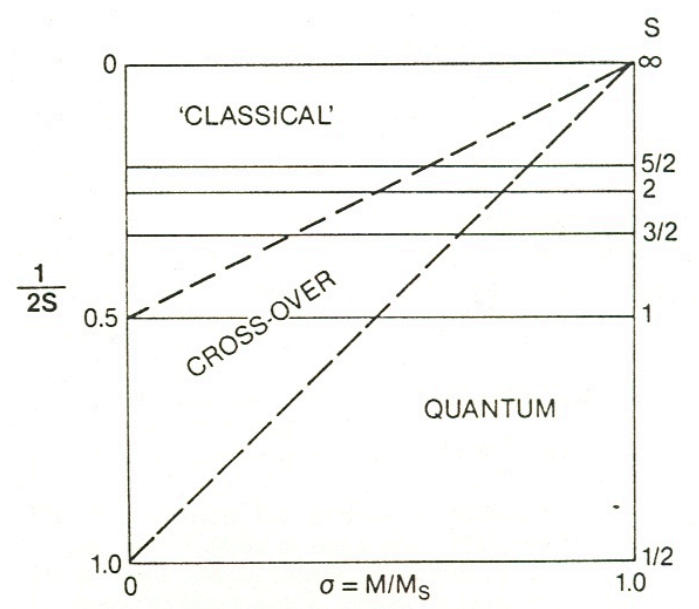

Figure 1. Quantum-classical crossover (schematic).

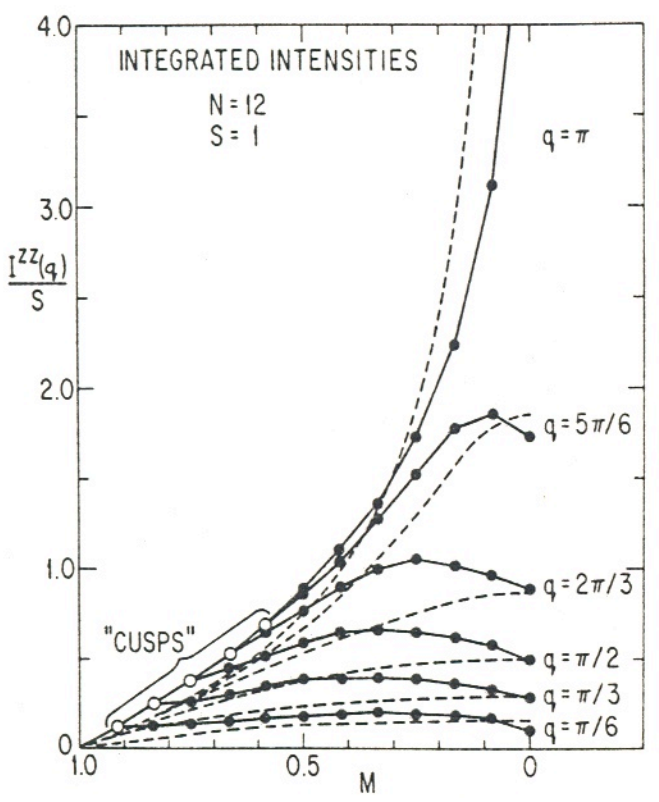

Figure 2. Quantum and classical features in the integrated intensity.

\section{The Haldane prediction}

Singlet-triplet energy gap excitation calculations for $S=1$ are consistent with previous numerical work $[3,4]$ in indicating a non-zero excitation gap in the thermodynamic limit. Such a gap, which does not occur for the exactly solvable $S=1 / 2$ system, is consistent with Haldane's predictions [1-4]. However, since the $N$-value large enough to indicate the correct asymptotic limiting behavior is in question [5], a Monte Carlo approach has been developed for $S=1, N$ up to 32 [3,6]. The 
Monte Carlo calculations agree with the exact finite-chain data out to $N=14$, and continue the exponential trend to a non-zero gap [6]. All indications are that the asymptotic $N$ region has been reached for the singlet-triplet "primary" excitation gap. In finite HBAFM's, the ground state $\left.\left(E_{0}\right)\right)$ is always a singlet and the first excited state a triplet $\left(E_{1}\right)$. Other low-lying excited states include a quintet $\left(E_{2}\right)$, a septet $\left(E_{3}\right)$, and other singlets. It has been verified that these other excitations also give rise to non-zero gaps in the thermodynamic limit [2]. These "higher gap" excitations are also important for the low-field behavior of the $T=0$ magnetization isotherm, which is well known to be a step function $[2,7]$. As illustrated in the inset to fig. 3, the location of the vertical portions of the steps is directly related to the higher excitations. Specifically, the first step distance is given by $H_{1}=E_{1}-E_{0}$, the second step by $H_{2}=E_{2}-E_{1}$, the third step by $H_{3}=E_{3}-E_{2}$, etc. In fig. 3, we show the step ratios $H_{2} / H_{1}$ and $H_{3} / H_{1}$ plotted for $S=1 / 2,1$ and $3 / 2$ for various $N$ values. The exact solution in the case of spin- $1 / 2$ indicates that the $T=0$ isotherm in the limit $\rightarrow \infty$ should be a straight line passing through the origin $[7,8]$. For finite spin- $1 / 2$ chains, this would imply, from simple geometric considerations, that $H_{2} / H_{1} \rightarrow 3, H_{3} / H_{1} \rightarrow 5$, and so on, in the thermodynamic limit. In fig. $3, H_{2} / H_{1}$ and $H_{3} / H_{1}$ are plotted versus $1 / N$, and a clear straight-line trend to the limiting values 3 and 5 , respectively, is apparent even for the smallest systems.

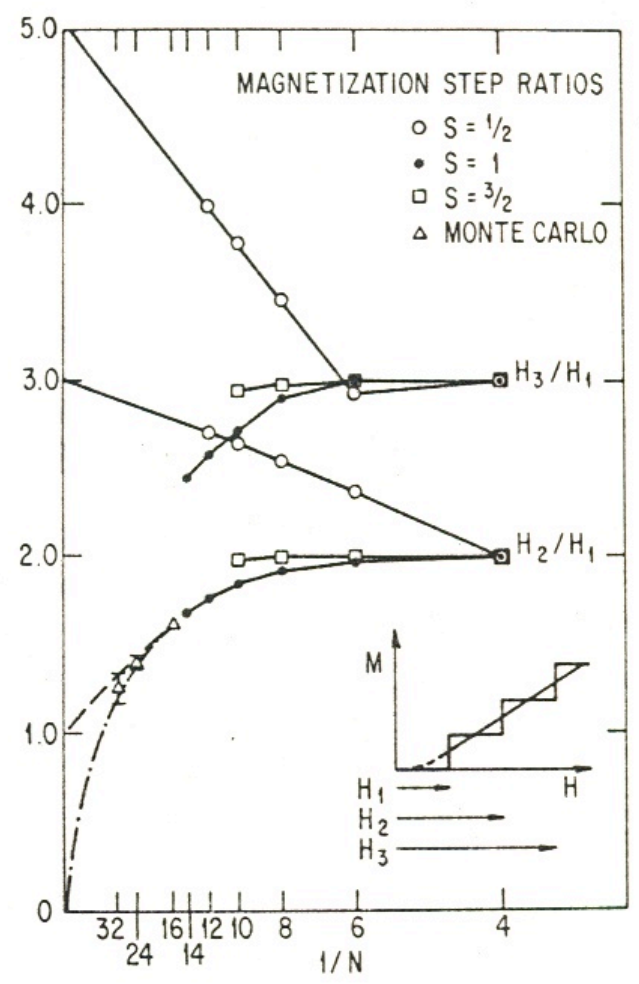

Figure 3. Magnetization step ratios for various spin values.

It is most interesting that the case of spin- 1 presents a complete contrast. Instead of rising, the step ratios $H_{2} / H_{1}$ and $H_{3} / H_{1}$ display a non-linear trend downwards. This is perhaps the most striking numerical evidence yet presented that spin- $1 / 2$ and spin- 1 chains behave very differently, as predicted by Haldane [1]. The prediction is made [6] that $E_{2}-E_{1} \sim 2\left(E_{1}-E_{0}\right)$, equivalent to a limiting value unity for the step ratio $H_{2} / H_{1}$. The actual Monte Carlo data points in fig. 3 indicate a trend to a value less than unity, perhaps even to zero. However, when uncertainty estimates are applied to the Monte Carlo data, a trend to unity is not excluded. In view of the fact that an $N \rightarrow \infty$ value of zero would have unusual implications, further extension and refinement of the Monte Carlo technique is underway. 
Finally we consider the case of spin-3/2. According to the predictions of Haldane [1], spin-3/2 should behave asymptotically in a manner similar to spin- $1 / 2$. This is not evident from the data extending to $N=10$ shown in fig. 3. No sign of an upward trend so apparent in the case of spin$1 / 2$ is observed, the data showing a slight tendency to decrease more reminiscent of spin- 1 . Two possibilities arise. One is that the true asymptotic behavior only sets in for much longer chains in the case of spin- $3 / 2$ than for spin- 1 and spin- $1 / 2$. The other is that spin- $1 / 2$ is an exactly solvable (integrable) case, differing in this respect from HBAFM's with $S \geq 1$ which therefore resemble each other rather than spin-1/2. Monte Carlo studies of the interesting case of spin- $3 / 2$ are therefore contemplated.

Acknowledgment: Support is acknowledged from the US N.S.F., grant numbers DMRS4061S6 and DMRSO-IOSI9, from NATO, from a Research Corporation grant, and from the SERC (UK) grant number GRID 4157. We have used a modified cmpj.sty style file.

\section{References}

1. F.D.M. Haldane, Phys. Lett. 93A (1983) 464; Phys. Rev. Lett. 50 (1983) 1153.

2. [21 J.B. Parkinson and J.C. Bonner, Phys. Rev. B 32 (1985) 4703.

3. J.B. Parkinson. J.C. Bonner. G. Müller. M.P. Nightingale and H.W.J. Blöte, J. Appl. Phys. 57 (1985) 3319.

4. R. Botet and R. Jullien, Phys. Rev. B 27 (1983) 613; M. Kolb. R. Botet and R. Jullien, J. Phys. A 16 (1983) L673.

5. J.C. Bonner and G. Müller, Phys. Rev. B 29 (1984) 5216.

6. M.P. Nightingale and H.W.J. Blöte, unpublished work.

7. J.C. Bonner and M.E. Fisher, Phys. Rev. 135 (1964) A614.

8. R.B. Griffiths, Phys. Rev. 133 (1968) A768. 